\title{
Increasing Physical Activity In Older Australians to Reduce Falls: A Program Evaluation
}

\author{
Kristy Robson \\ Charles Sturt University, krobson@csu.edu.au
}

Nazmul Ahasan

Murrumbidgee Local Health District NSW Australia, nazmul.ahasan@health.nsw.gov.au

Carly Barnes

Murrumbidgee Local Health District, NSW Australia, carly.barnes@health.nsw.gov.au

Kylie Murphy

Charles Sturt University, kmurphy@csu.edu.au

Rodney Pope

Charles Sturt University, rpope@csu.edu.au

Follow this and additional works at: https://nsuworks.nova.edu/ijahsp

Part of the Community Health and Preventive Medicine Commons, Physical Therapy Commons, and the Public Health Education and Promotion Commons

\section{Recommended Citation}

Robson K, Ahasan N, Barnes C, Murphy K, Pope R. Increasing Physical Activity In Older Australians to Reduce Falls: A Program Evaluation. The Internet Journal of Allied Health Sciences and Practice. 2021 Jan 01;19(3), Article 3.

This Manuscript is brought to you for free and open access by the College of Health Care Sciences at NSUWorks. It has been accepted for inclusion in Internet Journal of Allied Health Sciences and Practice by an authorized editor of NSUWorks. For more information, please contact nsuworks@nova.edu. 


\title{
Increasing Physical Activity In Older Australians to Reduce Falls: A Program Evaluation
}

\begin{abstract}
Purpose: As people age, they are at greater risk of injurious falls. Falling has a significant impact not only on the individual but also the wider community. Undertaking physical activity is effective in reducing the rate of falls in this population. Therefore, providing targeted education during group-based falls prevention programs may increase the awareness and amount of physical activity older people undertake to assist in reducing their risk of falling. Methods: A longitudinal cohort design involving a pre-post intervention survey was conducted over an eight-month period with community-dwelling older adults who participated in a fall-prevention program. Participants were $\mathrm{N}=161$ (123 female and 38 male), aged 65 years and over, with the most common age bracket being 75 to 84 years. Demographic information was collected at baseline. It included falls history and self-reported physical activity levels. Immediately post-intervention, self-reported changes in the awareness of the role of physical activity and awareness of falls risks were measured. Six months post-intervention, participants self-reported their physical activity levels and postprogram fall history. Results: An increase in awareness of the role of physical activity in reducing falls risk as well as falls risk factors was reported in most participants after completing the program. Despite this, only around a third of participants increased their physical activity levels during the six months after the program even though a decrease in falls rates was noted. Conclusions: The targeted education within the falls prevention program demonstrated an increase in awareness of falls risk factors and the importance of regular physical activity to minimise the risk of falling. However, this awareness did not seem to result in an increase in the amount of physical activity participants undertook after the program, even though falls rates across the participants reduced. Further research is needed to explore why older people who understand the benefits of undertaking regular physical activity did not increase their activity levels.
\end{abstract}

\section{Author Bio(s)}

Kristy Robson, $\mathrm{PhD}, \mathrm{MHSc}($ Education), DipHSc(Podiatry), is a registered podiatrist and academic in the School of Community Health at Charles Sturt University.

Nazmul Ahasan, BSc(with Honours), MSc(Biochemistry), MSc(Nutrition \& Dietetics), is the Falls Prevention Coordinator in the Directorate of Integrated Care and Allied Health Suite, Murrumbidgee Local Health District.

Carly Barnes is the Stepping On Falls Program Coordinator, Health Promotion Unit, Murrumbidgee Local Health District.

Kylie Murphy, PhD, BAppSc(PsychologyHons) BEd(Secondary), as an academic in the School of Community Health, Charles Sturt University

Rodney Pope, PhD, BAppSc(Phty), GradDipPyschStud, is a registered Physiotherapist and Professor of Physiotherapy in the School of Community Health, Charles Sturt University.

\section{Acknowledgements}

We would like to acknowledge Mark Filmer for his editorial assistance. 


\title{
1IJAHSP \\ The Internet Journal of Allied Health Sciences and Practice \\ Dedicated to allied health professional practice and education \\ Vol. 19 No. 3 ISSN 1540-580X
}

\section{Increasing Physical Activity in Older Australians to Reduce Falls: A Program Evaluation.}

\author{
Kristy Robson ${ }^{1}$ \\ Nazmul Ahasan² \\ Carly Barnes ${ }^{2}$ \\ Kylie Murphy ${ }^{1}$ \\ Rodney Pope ${ }^{1}$
}

1. Charles Sturt University

2. Murrumbidgee Local Health District

Australia

\begin{abstract}
Purpose: As people age, they are at greater risk of injurious falls. Falling has a significant impact not only on the individual but also the wider community. Undertaking physical activity is effective in reducing the rate of falls in this population. Therefore, providing targeted education during group-based falls prevention programs may increase the awareness and amount of physical activity older people undertake to assist in reducing their risk of falling. Methods: A longitudinal cohort design involving a pre-post intervention survey was conducted over an eight-month period with community-dwelling older adults who participated in a fallprevention program. Participants were $\mathrm{N}=161$ (123 female and 38 male), aged 65 years and over, with the most common age bracket being 75 to 84 years. Demographic information was collected at baseline. It included falls history and self-reported physical activity levels. Immediately post-intervention, self-reported changes in the awareness of the role of physical activity and awareness of falls risks were measured. Six months post-intervention, participants self-reported their physical activity levels and post-program fall history. Results: An increase in awareness of the role of physical activity in reducing falls risk as well as falls risk factors was reported in most participants after completing the program. Despite this, only around a third of participants increased their physical activity levels during the six months after the program even though a decrease in falls rates was noted. Conclusions: The targeted education within the falls prevention program demonstrated an increase in awareness of falls risk factors and the importance of regular physical activity to minimise the risk of falling. However, this awareness did not seem to result in an increase in the amount of physical activity participants undertook after the program, even though falls rates across the participants reduced. Further research is needed to explore why older people who understand the benefits of undertaking regular physical activity did not increase their activity levels.
\end{abstract}

Keywords: falls risk, older people, exercise, physical activity, falls prevention

(C) The Internet Journal of Allied Health Sciences and Practice, 2021 


\section{INTRODUCTION}

As people age, it is common to see reduced functional capacity with physiological and musculoskeletal decline. ${ }^{1}$ It is well evidenced that this type of age-related decline can significantly predispose older people to a greater risk of falling, with falls in the older person representing one of the leading causes of unintentional injury worldwide. ${ }^{2-6}$ Estimates suggest that one in three older people will fall every year, which can result in significant personal and economic impacts on the individual as well as the wider community.7,8 In addition, older adults who have experienced a fall are over $50 \%$ more likely to fall again within a year, potentially suffering from additional injuries that further reduce their mobility and independence. ${ }^{9,10}$ As such, the current rates of older people falling are concerning, especially given the proportion of people over 65 years of age will triple within the next 30 years. ${ }^{11}$ Therefore, instigating effective falls prevention strategies to reduce the risk of falling for older people living within the community is an urgent priority for health service providers and government organisations. ${ }^{8}$

The risk of falling in older people can be reduced, with evidence suggesting that physical activity, particularly activity that includes strength and balance components, is effective in preventing falls and maintaining mobility in older people living within the community. $8,12,13$ Several systematic reviews have shown that physical activity or exercise as part of a multifactorial approach or even as a single intervention can reduce both an individual's risk of falling and the rate of falling in older people. 8,12,13 Therefore, encouraging older people to participate in regular physical activity may have several benefits, including maintaining mobility levels and falls reduction as well as being a cost effective, population-wide approach to injury prevention. 8,12-14 However, it can be challenging to engage older people in undertaking and adhering to exercise programs, with an estimated $50 \%$ of older people worldwide considered to be physically inactive. 15,16 In addition, very few older adults undertake regular exercise that incorporates balance and strength at the recommended levels to prevent falls. ${ }^{17,18}$ Studies have shown that older people perceive several barriers to participating in physical activity, such as lack of support, chronic medical conditions, lack of motivation, or even ageist attitudes that encourage inactivity. 19,20 Some older people also perceive that their physical activity levels are adequate and that incorporating more balance and strength exercise or additional falls prevention strategies is not relevant to them. ${ }^{21-23}$

It is widely acknowledged that group-based falls prevention programs that incorporate strength and balance exercises reduce the risk of falls in older people.7,13,24,25 Group-based falls prevention programs also have the potential to increase understanding of the risks associated with falling as well as providing participants with targeted education on managing their own risk of falls. ${ }^{21,24}$ However, there is limited research on whether these programs can facilitate a greater understanding in older people of the need to undertake regular physical activity for general wellbeing over the long term, alongside assisting in reducing their risk of falling. One study evaluated whether a falls intervention program can change motivation for physical activity in older people, but they did not evaluate whether this motivation was sustained after the completion of the program ${ }^{21}$. Evidence also suggests that the benefits of the exercise component of these types of targeted programs can be lost if older people are not motivated to continue with ongoing physical activity over the long term. ${ }^{26}$ While another study evaluated whether older participants had any falls occurrences 14 months after completion of the fall program, but this evaluation did not explore whether participants continued with physical activity during this time ${ }^{24}$.

This study aimed to determine whether a community-based falls prevention program that was being implemented by a regional health service would facilitate a change in participants' awareness and involvement with physical activity in the long term, to assist them in being more physically active and reducing their risk of falling. Specifically, the study focused on addressing the following questions:

1) What was the self-perceived impact of undertaking a falls prevention program on participants' awareness of the risks associated with falls, including the role of physical activity in reducing falls risk?

2) What was the self-reported impact of the falls prevention program on participants' physical activity levels?

3) Did the falls prevention program impact on the rate of self-reported falls during the six-month period after completion?

\section{METHODS}

\section{Study Design}

The community-based falls prevention program was delivered across a large regional area within Australia. It was run in a small group format in a variety of geographic locations. It involved a one-hour session face to face for seven weeks with older people aged 65 years and older, who were either self-referred or referred into the program by a health professional and who were at risk of falling. Each week the program consisted of a 60-minute exercise component and a 60-minute education component that focused on increasing participant's awareness of the risks associated with falling and strategies to reduce these risks, including undertaking regular and ongoing physical activity. The present study involved a longitudinal cohort design involving repeated measures obtained through a three-phase survey approach over a period of 8 months. Paper-based survey questionnaires were provided to participants at the beginning of the community-based falls prevention program in week 1 (baseline survey) and again at the end of the program in week 7 (post program survey). Participants were then contacted by telephone six months after completing the

๑ The Internet Journal of Allied Health Sciences and Practice, 2021 
program to undertake the final survey (six-month follow-up survey). The study was opportunistic, seeking to determine outcomes and factors contributing to outcomes of the community-based program, which was being implemented by the health service, independent of the research team.

\section{Study Sample and Procedures}

The study participants were community-dwelling older people aged 65 years and older who had either experienced a fall during the previous six months or had identified risk factors for falls, including fear of falling, and so were participating in the communitybased falls prevention program. All older people who participated in the regional community-based falls prevention program were invited to participate in the study, with information sheets provided when individuals expressed an interest in attending the program. Participants who consented to being involved in the study were provided with a copy of the survey questionnaire and a selfaddressed envelope so they could answer both the pre- and post-program survey questionnaires without the program facilitators having access to the data. All sealed envelopes were collected by the program facilitators at the time of survey completion (week 1 and 7 ) and forwarded to the research team for analysis.

Across the data collection period, 192 participants agreed to be involved in the study. Of the 192 participants, 161 older people at least partially completed the baseline survey (in Week 1), 135 at least partially completed both the baseline survey and the post program survey (in Week 7 ), and 84 participants completed all three surveys. Participants were allocated an individual participant number so that data between the surveys could be matched.

Demographic and falls information for participants involved at each time point can been seen in Table 1.

Table 1. Participant demographic and falls data.

\begin{tabular}{|c|c|c|c|}
\hline & Gender & Age & Falls history at baseline \\
\hline $\begin{array}{l}\text { Completed } \\
\text { Baseline Survey } \\
\text { (161) }\end{array}$ & $\begin{array}{l}\text { Female }-76 \%(n=123) \\
\text { Male }-24 \%(n=38)\end{array}$ & $\begin{array}{l}65 \text { to } 74-22 \%(n=35) \\
75 \text { to } 84-40 \%(n=65) \\
>85-21 \%(n=33) \\
\text { No response }-17 \%(n=28)\end{array}$ & $\begin{array}{l}\text { Yes }-32 \%(n=52) \\
\text { No }-51 \%(n=82) \\
\text { No response }-17 \%(n=27)\end{array}$ \\
\hline $\begin{array}{l}\text { Completed } \\
\text { Baseline and Post } \\
\text { Program Survey } \\
\text { (135) }\end{array}$ & $\begin{array}{l}\text { Female }-78 \%(n=106) \\
\text { Male }-22 \%(n=29)\end{array}$ & $\begin{array}{l}65 \text { to } 74-19 \%(n=26) \\
75 \text { to } 84-40 \%(n=54) \\
>85-20 \%(n=27) \\
\text { No response }-21 \%(n=28)\end{array}$ & $\begin{array}{l}\text { Yes }-27 \%(n=36) \\
\text { No }-53 \%(n=72) \\
\text { No response }-20 \%(n=27)\end{array}$ \\
\hline $\begin{array}{l}\text { Completed all } 3 \\
\text { Surveys } \\
\text { (84) }\end{array}$ & $\begin{array}{l}\text { Female }-83 \%(n=70) \\
\text { Male }-17 \%(n=14)\end{array}$ & $\begin{array}{l}65 \text { to } 74-20 \%(n=17) \\
75 \text { to } 84-42 \%(n=35) \\
>85-19 \%(n=16) \\
\text { No response }-19 \%(n=16)\end{array}$ & $\begin{array}{l}\text { Yes }-30 \%(n=25) \\
\text { No }-52 \%(n=44) \\
\text { No response }-18 \%(n=15)\end{array}$ \\
\hline
\end{tabular}

$\mathrm{n}=$ number of participants

\section{Data Collection}

\section{Baseline Survey}

Demographic information regarding falls and physical activity information was collected from all participants at baseline (survey 1) and included age, gender, falls history (pre-intervention fall occurrence in the 6 months prior to the intervention) and information relating to self-reported current physical activity levels. Physical activity was defined as any type of deliberate exercise the older person was undertaking, including walking, sporting activities, gardening, or structured exercise programs (individually or in a group setting). Participants were asked to self-report that average amount of time per week they were undertaking physical activity in the baseline survey. In subsequent surveys, participants were asked to self-report whether they perceived that had increased, maintained, or decreased the amount of physical activity they were undertaking at baseline. Each participant's perceived understanding of the role that physical activity plays in promoting general well-being was also ascertained in survey 1.

\section{Outcome Measures / Post Program Survey}

The following data was collected to evaluate the influence of the program on the intended outcomes that mediated longer term impacts of the program.

Change in physical activity awareness. The self-perceived effect of the program on participants' awareness of the importance of physical activity was assessed categorically by one item in the post-intervention survey: "Do you believe the Program has made you more aware of the role physical activity plays in reducing your risk of falls?" Response options were "Yes," "Unsure," or "No, I was aware before I started the program." 
Change in risk factor awareness. The self-perceived effect of the program on participants' awareness of risk factors for falling was assessed categorically by one item in the post-intervention survey: "Do you believe the Program has made you more aware of your risks associated with falling?" Response options were "Yes," "Unsure," or "No, I was aware before I started the program."

\section{Impact Measures / Six-month Follow up Survey}

The following data was collected to evaluate the effects of the program in relation to the intended longer-term impacts.

Change in physical activity level. Participants were asked in the six-month post-intervention survey whether their activity level had increased, stayed the same, or decreased compared to before the program. This data was recorded categorically.

Post-program fall occurrence. Any occurrence of a fall in the six-month period following the program was self-reported categorically: 'Yes' or "No."

\section{Data Analysis}

The following program logic guided the statistical analysis. ${ }^{27}$ The two awareness variables were treated as program outcome variables. The physical activity level change and post-program fall status were viewed as program impact variables (the assumed impact of the outcome variables). All these variables were analysed descriptively with percentages. To test the assumed program logic, the relationship between each outcome variable and each impact variable was tested using the chi square test for independence ${ }^{28}$. Alpha was set at .05 .

The participants' data were then grouped by pre-program fall status for sub-group analysis, with sub-groups established based on the status of each participant as: "with pre-program fall" or "without pre-program fall." The relationships between each outcome and impact variable and pre-program fall status were examined by descriptive cross-tabulation.

\section{RESULTS}

Pre-Program Physical Activity and Fall Status

Among the 161 participants who completed the baseline survey, there was variability in the self-reported pre-program physical activity levels, with $18.6 \%$ engaged in less than one hour per week, $22.9 \%$ engaged in one to two hours, $19.8 \%$ engaged in two to four hours, $29.1 \%$ engaged in more than four hours per week, and $9.3 \%$ providing no response for this question. For the six months preceding their commencement of the program, $51 \%$ self-reported no pre-program fall and $32 \%$ indicated they had experienced at least one fall; $17 \%$ did not respond to this question.

\section{Outcomes}

\section{Change in Physical Activity Awareness}

Of the 135 participants who responded to this survey question, 129 (95.6\%) reported an increase in physical activity awareness, $1(0.7 \%)$ reported being unsure about whether there was an increase, and $5(3.7 \%)$ reported no change.

\section{Change in Risk-Factor Awareness}

Regarding risk awareness, of the 135 participants who answered the question, $118(86.8 \%)$ self-reported an increase in risk awareness due to the program, no participants reported being unsure, and $18(13.2 \%)$ reported no change in their risk awareness.

\section{Impact Results}

\section{Change in Physical Activity Level}

Overall, of the 84 participants who completed the relevant question from survey three, $26(31 \%)$ self-reported an increase in physical activity at the six-month follow-up survey compared to their reported levels in the baseline survey, $36(42.9 \%)$ reported no change in their physical activity, and $22(26.2 \%)$ reported a decrease in their physical activity. The most common reasons for decreasing physical activity reported by participants were health issues, time constraints, and lack of motivation. Of the participants that answered both questions and self-reported at baseline undertaking either between two and four hours of exercise per week, $78.5 \%$ indicated they had increased their levels of physical activity over the 6 month period following the program, and of those who reported undertaking over 4 hours of exercise per week, $65 \%$ maintained their levels of physical activity.

\section{Post-Program Fall Status}

Among the 84 participants who completed both the baseline survey and six-month follow up survey, a total of 71 participants $(84.5 \%)$ responded to the falls question and reported no falls during the six-month period after their completion of the program, compared to $63.8 \%$ for the six-month period preceding participation in the program. More detailed post-program falls data is presented in Table 2 below. 


\section{Relationships Between the Outcome and Impact Variables Physical Activity Awareness and Impact Variables}

Of the 76 participants who reported an increase in physical activity awareness and answered whether they had changed their physical activity during the six months post intervention, $23(30.3 \%)$ reported an increase in their physical activity level, $33(43.4 \%)$ reported no change, and $20(26.3 \%)$ reported a decrease in their physical activity level. A chi square test for independence revealed a small to medium effect size (Cramer's $V=.15$ ) for the relationship between increased physical activity awareness and increased physical activity level; and statistical significance was not reached $(p=.48)$.

Of the 77 participants who reported an increase in physical activity awareness and responded to the post-program fall question, $65(84.4 \%)$ reported no fall event during the six months after the program and $12(15.6 \%)$ reported experiencing a fall. A total of 5 participants reported no increase in their awareness (4) or that they were unsure (1). All those participants reported no fall event post-program. These results represent a small to medium effect size (Cramer's $V=.11)$ for the relationship between increased physical activity awareness and post-program fall status; and statistical significance was not reached $(p=.63)$.

\section{Risk-Factor Awareness and Impact Variables}

Of the 72 participants who reported an increase in risk awareness and answered the physical activity level-change question, 25 (34.7\%) reported an increase in their physical activity level, 29 (40.3\%) reported no change, and 18 (25\%) reported a decrease in physical activity level. Based on a chi square test for independence, these results represent a small to medium effect size (Cramer's $V=.18$ ) for the relationship between increased risk awareness and increased physical activity level; and statistical significance was not reached $(p=.27)$.

Of the 73 participants who reported an increase in risk awareness and responded to the post-program fall question, 61 (83.6\%) reported having no falls during the six months post-program and $12(16.4 \%)$ reported experiencing a fall. These results represent a small to medium effect size (Cramer's $V=.15$ ) for the relationship between increased risk awareness and post-program fall status; and statistical significance was not reached $(p=.17)$.

\section{Relationship Between Pre-Program Fall-Status and Outcomes and Impacts}

Table 2 shows the proportions of participants who achieved each target outcome and target impact. These results show that despite negligible differences between the target outcome rates of participants with a history of falls versus no history of falling (in the six months before the program), differences were observed between those two sub-groups in relation to the target impact rates. As can be seen in Table 2, noticeably more participants who experienced a fall before the program self-reported an increase in their physical activity (37.9\%) when compared with participants who had no falls history before the program (27.3\%). However, this relationship was not statistically significant (Cramer's $V=.11, p=.60$ ). Additionally, more participants with a pre-program fall selfreported experiencing a fall in the six months following the program $(27.6 \%)$ when compared with participants who had not experienced a pre-program fall $(10.7 \%)$. Chi square analysis revealed that this relationship was statistically significant $(p=.047)$ and represents a medium-sized effect (Cramer's $V=.22$ ). However, while being fall-free before the program was associated with fewer falls after the program, it is noteworthy that only $27.6 \%$ of those participants who had experienced a fall during the six months preceding the program reported falling in the equivalent six-month period after completing the program.

Table 2. Target outcome and target impact comparisons based on pre-program fall-status.

\begin{tabular}{|c|c|c|c|c|}
\hline es & \multicolumn{2}{|c|}{ Target Outcomes } & \multicolumn{2}{|c|}{ Target Impacts } \\
\hline $\begin{array}{l}\text { Pre-program fall } \\
\text { status }\end{array}$ & $\begin{array}{l}\text { Increased physical } \\
\text { activity awareness }\end{array}$ & $\begin{array}{l}\text { Increased risk } \\
\text { awareness }\end{array}$ & $\begin{array}{c}\text { Increased physical } \\
\text { activity }\end{array}$ & $\begin{array}{c}\text { No fall } \\
\text { post-program }\end{array}$ \\
\hline $\begin{array}{l}\text { With pre-program } \\
\text { fall }\end{array}$ & $95 \% n=38$ & $90.0 \% n=36$ & $37.9 \% n=11$ & $72.4 \% n=21$ \\
\hline $\begin{array}{l}\text { Without pre- } \\
\text { program fall }\end{array}$ & $96.3 \% n=79$ & $88.0 \% n=73$ & $27.3 \% n=15$ & $89.3 \% n=50$ \\
\hline
\end{tabular}

(๖ The Internet Journal of Allied Health Sciences and Practice, 2021 


\section{DISCUSSION}

This study examined the self-perceived effects of a community-based falls prevention program on older adults' self-reported physical activity awareness, falls-risk awareness, engagement in physical activity, and post-program fall status. Awareness of the role of physical activity and awareness of falls risk factors were viewed as outcome variables (in a post program survey), and engagement in physical activity and post-program falls rates were viewed as impact variables (in a survey six months after program completion). Given that the demographics of the participants in all three surveys were similar, it is reasonable to assume that there was no attrition bias.

Regarding achieving the desired impacts of the program, this study found that only around a third of participants increased their physical activity during the six months following the program despite almost all participants being more aware of the role physical activity can play in reducing falls and increasing general wellbeing and mobility. Interestingly, most participants who did increase their levels of physical activity were already undertaking a reasonable amount physical activity before the program. This may indicate that older people who are already motivated to be active are more likely to engage in additional physical activity when encouraged. Previous research evidence has suggested that older adults who habitually undertake physical activity are more likely to seek out opportunities to include further physical activity in their daily activities. ${ }^{29,30}$

While most participants did not report an increase in their physical activity level following program completion, they also reported not falling during the six months following the program. Reduced falls-rates were observed, with only $27.6 \%$ of participants who experienced a fall before the program subsequently falling during the equivalent post program period, despite their increased risk of falling, and overall fall rates reducing from $85 \%$ in the 6 months preceding the program to $64 \%$ in the 6 months following the program - a relative reduction in the overall fall rate of $33 \% .{ }^{9}$ These results indicate that this falls prevention program may have contributed to the reduced the number of falls experienced by older persons who were at risk of subsequent falling, consistent with the current literature. ${ }^{24,31}$

A large proportion of participants in this study self-reported an increased awareness of the importance of physical activity in preventing falls as well as their personal risks associated with falling. However, while these two outcome variables were assumed to be mechanisms that might assist in improving the two impact variables (increased physical activity and reduced post-program falls), neither of these outcome variables impacted significantly on either of the impact variables. Also, this study found that a greater number of participants achieved the fall-free impact target when compared with the physical activity impact target. Understanding the importance of increasing physical activity did not translate into a rise in regular physical activity, particularly for participants who were undertaking minimal amounts of physical activity before the program.

In addition, a reduction in rates of self-reported falls events occurred even though there was no significant change in physical activity levels. For participants in this program, increasing the awareness of falls risks may have been a greater protector from falling than the efforts to raise their awareness of the need for increased physical activity, despite strong evidence that physical activity has the potential to significantly reduce falls in older people.25,32 This is a complex area and possibly participants in this study were undertaking other modifications in their daily lives to further reduce their risk of falling other than increasing their physical activity, based on their increased awareness of falls related risk factors. There are also several barriers commonly reported in the literature as to why older people do not maintain the recommended level of physical activity, such as health concerns, attitudes, and lack of motivation or opportunity. ${ }^{33-35}$ Any or all of these factors may have contributed to the results observed in this study.

The study also demonstrated that some participants still fell during the six months after the program despite attending the falls prevention program. Given the current evidence on the importance of regular physical activity for older people, it is clear that changing behaviour in this area is an appropriate strategy to be included into group-based fall prevention programs, not only to reduce the risk of falls but also for maintaining mobility and independence. However, to make this aspect more successful in translating knowledge into action, research suggests that strategies that incorporate behavioural change theory should be embedded into educational sessions in order to encourage greater uptake of physical activity, particularly for older people who are currently inactive. ${ }^{21,36}$

\section{Limitations}

There were several limitations in this study. Firstly, the small final numbers of participants and loss to follow up in each of the subgroups may have impacted on the overall results. Secondly, all the outcome and impact variables measured in this study were self-reported. In addition, the two awareness variables were not self-reported both pre- and post-program; instead, participants were asked to self-report whether they believed their awareness had been improved by the program. This relied on participant memory and may not have rendered accurate responses. Thirdly, while physical activity levels were self-reported pre-program, physical activity levels were not measured again post-program due to the study being a community-based program and limited

( The Internet Journal of Allied Health Sciences and Practice, 2021 
scope to be able to undertake further validated measures; instead participants were required to self-report whether they believed their physical activity levels had increased, remained the same, or decreased. On this basis, additional research is needed to further study these important outcomes, impacts and potentially mediating relationships relating to physical activity and falls in older people using validated measures.

\section{CONCLUSION}

Falls in older adult populations are associated with significant social and economic issues worldwide. Alongside this, half of older people are not undertaking the recommended level of regular physical activity. Studies have shown that increasing physical activity can have a protective effect on falls rates and community-based falls prevention programs provide an opportunity to interact with older people and educate them on the role of physical activity in reducing falls and maintaining overall wellbeing. However, despite the current study demonstrating an increase in participant's awareness of falls risk factors and strategies to reduce falls, including the role of physical activity, this did not translate to a self-reported increase in the amount of physical activity participants undertook after the program, even though falls rates across the participants reduced. Further research is needed to explore why participants in this regional area who increased their understanding of undertaking regular physical activity did not improve their exercise levels and the importance of incorporating behavioural change approaches in participant education in this area is again highlighted.

\section{DECLARATIONS}

\section{Ethical Approval and Consent to Participate}

The Greater Western Area Health Service Human Research Ethics Committee approved this study. (HREC reference number: LNR/14/GWAHS/48). All participants gave written informed consent to participate before data collection.

\section{Consent for Publication}

No individual participant data is presented in this study.

\section{Availability of Data and Materials}

The datasets used and/or analysed during the current study are available from the corresponding author on reasonable request and subject to the Human Research Ethics Committee that approved the study.

\section{Competing Interests}

Nil competing interests to declare.

\section{Funding}

Funding was provided by the Murrumbidgee Local Health District to support a research assistant to enter project data for analysis.

\section{Author's Contributions}

Research protocol was conceived by KR, NA, CB and RP. NA and CB designed the survey tool and telephone questions, CB collected the data, KR, KM and RP analysed the data. KR and KM drafted the manuscript with all authors providing critical review and approving the final version.

\section{Acknowledgements}

We would like to acknowledge Mark Filmer for his editorial assistance.

\section{REFERENCES}

1. Roberts S, Colombier P, Sowman A, Mennan C, Rölfing JH, Guicheux J, Edwards JR. Ageing in the musculoskeletal system: cellular function and dysfunction throughout life. Acta Orthop. 2016 Dec 16;87(sup363):15-25.

2. Houry D, Florence $C$, Baldwin G, Stevens J, McClure R. The CDC Injury Center's response to the growing public health problem of falls among older adults. Am J. Lifestyle Med. 2016 Jan;10(1):74-7.

3. Sawers A, Pai YC, Bhatt T, Ting LH. Neuromuscular responses differ between slip-induced falls and recoveries in older adults. J Neurophysiol. 2016 Nov 2;117(2):509-22.

4. Ambrose AF, Paul G, Hausdorff JM. Risk factors for falls among older adults: a review of the literature. Maturitas. 2013 May 1;75(1):51-61.

5. Deandrea S, Lucenteforte E, Bravi F, Foschi R, La Vecchia C, Negri E. Risk factors for falls in community-dwelling older people:"a systematic review and meta-analysis". Epidemiology. 2010 Sep 1:658-68. 
6. World Health Organization. Falls fact sheet. Diakses dari: http://www. who. int/mediacentre/factsheets/fs344/en/on November. 2017 Sep;29:2017.

7. Sherrington, Catherine, and Anne Tiedemann. Physiotherapy in the prevention of falls in older people. J Physiother. 61, no. 2 (2015):54-60.

8. Sherrington C, Michaleff ZA, Fairhall N, Paul SS, Tiedemann A, Whitney J, Cumming RG, Herbert RD, Close JC, Lord SR. Exercise to prevent falls in older adults: an updated systematic review and meta-analysis. Br J Sports Med. 2017 Dec 1;51(24):1750-8.

9. O'Loughlin JL, Robitaille Y, Boivin JF, Suissa S. Incidence of and risk factors for falls and injurious falls among the community-dwelling elderly. Am J Epidemiol. 1993 Feb 1;137(3):342-54.

10. Todd C, Skelton D. What are the Main Risk Factors for Falls Amongst Older People and what are the Most Effective Interventions to Prevent These Falls? World Health Organization; 2004.

11. Beard JR, Officer AM, Cassels AK. The World Report on Ageing and Health. Gerontologist. 2016;56 Suppl 2:S163S166. doi:10.1093/geront/gnw037.

12. Michael YL, Lin JS, Whitlock EP, et al. Interventions to Prevent Falls in Older Adults: An Updated Systematic Review. Agency for Healthcare Research and Quality (US), Rockville (MD); 2010.

13. Gillespie LD, Robertson MC, Gillespie WJ, et al. Interventions for preventing falls in older people living in the community. Cochrane Database Syst Rev. 2012;(9):CD007146. Published 2012 Sep 12. doi:10.1002/14651858.CD007146.pub3).

14. Davis JC, Robertson MC, Ashe MC, Liu-Ambrose T, Khan KM, Marra CA. Does a home-based strength and balance programme in people aged $\geq 80$ years provide the best value for money to prevent falls? A systematic review of economic evaluations of falls prevention interventions. Br J Sports Med. 2010 Feb 1;44(2):80-9.

15. Franco MR, Howard K, Sherrington C, Ferreira PH, Rose J, Gomes JL, Ferreira ML. Eliciting older people's preferences for exercise programs: a best-worst scaling choice experiment. J Physiother. 2015 Jan 1;61(1):34-41.

16. Hallal PC, Andersen LB, Bull FC, et al. Global physical activity levels: surveillance progress, pitfalls, and prospects. Lancet. 2012;380(9838):247-257. doi:10.1016/S0140-6736(12)60646-1

17. Kraschnewski JL, Sciamanna CN, Ciccolo JT, Rovniak LS, Lehman EB, Candotti C, Ballentine NH. Is exercise used as medicine? Association of meeting strength training guidelines and functional limitations among older US adults. Prev Med. 2014 Sep 1;66:1-5.

18. Vezina JW, Der CA, Greenberg E, Kurka J. Sociodemographic correlates of meeting US Department of Health and Human Services muscle strengthening recommendations in middle-aged and older adults. Prev Chronic Dis 2014;11:E162.

19. Brawley LR, Rejeski WJ, King AC. Promoting physical activity for older adults: the challenges for changing behavior. Am J Prev Med. 2003 Oct 1;25(3):172-83.

20. Hardy S, Grogan S. Preventing disability through exercise: investigating older adults' influences and motivations to engage in physical activity. J Health Psychol. 2009 Oct;14(7):1036-46.

21. McMahon SK, Wyman JF, Belyea MJ, Shearer N, Hekler EB, Fleury J. Combining motivational and physical intervention components to promote fall-reducing physical activity among community-dwelling older adults: A feasibility study. Am J Health Promot. 2016 Nov;30(8):638-44.

22. Robson K, Coyle J, Pope R. Exploration of older people's perceptions of behavioural factors associated with falls. Age Ageing. 2018 Apr 12;47(5):734-40.

23. Yardley L, Bishop FL, Beyer N, Hauer K, Kempen GI, Piot-Ziegler C, Todd CJ, Cuttelod T, Horne M, Lanta K, Holt AR. Older people's views of falls-prevention interventions in six European countries. The Gerontologist. 2006 Oct 1;46(5):650-60.

24. Clemson L, Cumming RG, Kendig H, Swann M, Heard R, Taylor K. The effectiveness of a community-based program for reducing the incidence of falls in the elderly: A randomized trial. J Am Geriatr Soc. 2004 Sep;52(9):1487-94.

25. Sherrington C, Tiedemann A, Fairhall N, Close JC, Lord SR. Exercise to prevent falls in older adults: an updated metaanalysis and best practice recommendations. New South Wales Public Health Bulletin. 2011 Jun 23;22(4):78-83.

26. Close JC, Lord SL, Menz HB, Sherrington C. What is the role of falls? Best Pract Res Clin Rheumatol. 2005 Dec 1;19(6):913-35.

27. Rogers PJ. Logic models. In S. Mathison (Ed.), Encyclopedia of evaluation. Thousand Oaks, CA: Sage Publications Inc. 2005. doi: 10.4135/9781412950558

28. Salkind NJ. Encyclopedia of research design. Thousand Oaks, CA: SAGE Publications, Inc. 2010. doi: $10.4135 / 9781412961288$

29. Lally P, Van Jaarsveld CH, Potts HW, Wardle J. How are habits formed: Modelling habit formation in the real world. Eur J Soc Psychol. 2010 Oct;40(6):998-1009. 
30. Sparling PB, Howard BJ, Dunstan DW, Owen N. Recommendations for physical activity in older adults. BMJ. 2015 Jan 21;350:h100.

31. Skelton D, Dinan S, Campbell M, Rutherford O. Tailored group exercise (Falls Management Exercise-FaME) reduces falls in community-dwelling older frequent fallers (an RCT). Age Ageing. 2005 Nov 1;34(6):636-9.

32. Hamed, A., Bohm, S., Mersmann, F. et al. Follow-up efficacy of physical exercise interventions on fall incidence and fall risk in healthy older adults: a systematic review and meta-analysis. Sports Med - Open 4, 56 (2018). https://doi.org/10.1186/s40798-018-0170-z

33. Finnegan S, Bruce J, Seers K. What enables older people to continue with their falls prevention exercises? A qualitative systematic review. BMJ open. 2019 Apr 1;9(4):e026074.

34. Stathi A, McKenna J, Fox KR. Processes associated with participation and adherence to a 12-month exercise programme for adults aged 70 and older. J Health Psychol. 2010 Sep;15(6):838-47.

35. De Groot GC, Fagerström L. Older adults' motivating factors and barriers to exercise to prevent falls. Scand J Occup Ther. 2011 Jun 1;18(2):153-60.

36. McMahon SK, Lewis B, Oakes JM, Wyman JF, Guan W, Rothman AJ. Assessing the effects of interpersonal and intrapersonal behavior change strategies on physical activity in older adults: a factorial experiment. Ann Behav Med. 2017 Feb 10;51(3):376-90. 